\title{
Atuação dos enfermeiros no pré-natal a gestantes usuárias de álcool
}

Nursing prenatal care for pregnant women who use alcohol

Atención prenatal de enfermeira para mujeres embarazadas que consumen alcohol

Sônia Maria Alves de Paiva

ORCID: https://orcid.org/0000-0001-7918-3855 Escola Técnica de Centro Paula Souza, Brasil E-mail: soniaenf@hotmail.com

Ana Vitória Lima de Souza

ORCID: https://orcid.org/0000-0002-0623-6001

Santa Casa de Poços de Caldas, Brasil

E-mail: ana.lima92@ hotmail.com

Márcia Aparecida Ferreira de Oliveira ORCID: https://orcid.org/0000-0002-1069-8700

Universidade de São Paulo, Brasil E-mail: marciaap@usp.br

Júlia Carolina de Mattos Cerioni Silva ORCID: https://orcid.org/0000-0001-5367-4197

Universidade de São Paulo, Brasil E-mail: jucarol80@usp.br

Caroline Balan

ORCID: https://orcid.org/0000-0002-8908-6669

Universidade de São Paulo, Brasil E-mail: caroline.ballan@usp.br

Ana Victória Calado Godoy Carlos de Lima ORCID: https://orcid.org/0000-0002-8112-1001

Universidade de São Paulo, Brasil E-mail: lima.victoria@usp.br

Gabriella de Andrade Boska

ORCID: https://orcid.org/0000-0002-5827-6486

Universidade de São Paulo, Brasil E-mail: gabriellaboska@usp.br

Maria Regina Camargo Ferraz Souza ORCID: https://orcid.org/0000-0002-7197-1846

Universidade de São Paulo, Brasil E-mail: mrcferraz@usp.br

Priscilla de Oliveira Luz

ORCID: https://orcid.org/0000-0003-0120-1667

Universidade de São Paulo, Brasil E-mail: pris_luz@usp.br

Heloísa Garcia Claro

ORCID: https://orcid.org/0000-0003-1504-7074

Universidade Estadual de Campinas, Brasil

E-mail: clarohg@unicamp.br

Rosana Ribeiro Tarifa

ORCID: https://orcid.org/0000-0002-5785-4504 Faculdade Anhanguera de Sumaré, Brasil

E-mail: rosanart85@hotmail.com

\section{Resumo}

Objetivo: identificar o conhecimento dos enfermeiros sobre a Síndrome Alcoólica Fetal (SAF) e as ações promovidas por estes no pré-natal de gestantes usuárias de álcool nas Unidades Básicas de Saúde (UBS). Método: pesquisa com abordagem qualitativa, de caráter descritivo e exploratório, desenvolvida em UBSs, situadas em um município do interior de Minas Gerais. Os dados foram coletados por meio de entrevistas e, para a interpretação, utilizou-se a técnica de análise de conteúdo. Resultados: onze enfermeiros participaram deste estudo. Das informações analisadas, emergiram três categorias temáticas. Categoria Temática 1: falta conhecimento sobre a Síndrome Alcoólica Fetal; Categoria temática 2: estratégias utilizadas pelos enfermeiros no atendimento às gestantes usuárias de álcool; e, Categoria temática 3: dificuldades em se realizar a referência e contrarreferência na rede de atenção à saúde (RAS). Conclusão: Conclui-se que os enfermeiros possuem pouco conhecimento no que se refere aos efeitos teratogênicos do álcool, suas ações se baseiam em realizar busca ativa de gestantes usuárias de álcool, na tentativa de estabelecer o vínculo terapêutico. Dentre as dificuldades com relação à temática, a que mais impacta o processo assistencial é a inexistência de fluxo pactuado que respeite as obrigações pertinentes a referência e contrarrefência na rede de atenção 
à saúde. A busca por aprimoramento profissional na área da Saúde da Mulher e Saúde Mental, bem como o estabelecimento de um fluxo assistencial adequado na rede, asseguraria uma atenção integral à gestante e ao recémnascido.

Palavras-chave: Enfermagem; Gravidez; Alcoolismo; Síndrome alcoólica fetal; Saúde mental.

\begin{abstract}
Objective: to identify nurses' knowledge about Fetal Alcohol Syndrome (FAS) and the actions promoted by them in the prenatal care of pregnant women who are alcohol users in Basic Health Units (BHU). Method: A qualitative, descriptive and exploratory research was carried out in UBS, located in a city in the interior of Minas Gerais. Data were collected through interviews and, for interpretation, the content analysis technique was used. Results: eleven nurses participated in this study. From the information analyzed, three thematic categories emerged. Thematic Category 1: lack of knowledge about Fetal Alcohol Syndrome; Thematic Category 2: strategies used by nurses in the care of alcohol-using pregnant women; and, Thematic Category 3: difficulties in making reference and counterreference in the health care network (RAS). Conclusion: It was concluded that nurses have little knowledge regarding the teratogenic effects of alcohol, and their actions are based on actively searching for pregnant alcohol users in an attempt to establish the therapeutic bond. Among the difficulties related to the theme, the one that most impacts the assistance process is the lack of an agreed flow that respects the obligations related to reference and counter-reference in the health care network. The search for professional improvement in Women's Health and Mental Health, as well as the establishment of an adequate flow of care in the network, would ensure integral care to the pregnant woman and the newborn.
\end{abstract}

Keywords: Nursing; Pregnancy; Alcoholism; Fetal alcohol syndrome; Mental health.

\begin{abstract}
Resumen
Objetivo: identificar el conocimiento de las enfermeras sobre el Síndrome Alcohólico Fetal (SAF) y las acciones promovidas por ellas en el cuidado prenatal de las gestantes consumidoras de alcohol en las Unidades Básicas de Salud (UBS). Método: investigación con enfoque cualitativo, de carácter descriptivo y exploratorio, desarrollada en UBS situadas en un municipio del interior de Minas Gerais. Los datos se recopilaron mediante entrevistas y, para su interpretación, se utilizó la técnica de análisis de contenido. Resultados: once enfermeras participaron en este estudio. De la información analizada surgieron tres categorías temáticas. Categoría Temática 1: desconocimiento del Síndrome Alcohólico Fetal; Categoría Temática 2: estrategias utilizadas por las enfermeras en la atención a las gestantes consumidoras de alcohol; y, Categoría Temática 3: dificultades para hacer referencia y contrarreferencia en la red de atención a la salud (RAS). Conclusión: Se concluyó que las enfermeras tienen poco conocimiento sobre los efectos teratogénicos del alcohol, y sus acciones se basan en la búsqueda activa de embarazadas consumidoras de alcohol en un intento de establecer el vínculo terapéutico. Entre las dificultades relacionadas con el tema, la que más impacta en el proceso asistencial es la falta de un flujo consensuado que respete las obligaciones relacionadas con la referencia y contrarreferencia en la red asistencial. La búsqueda del perfeccionamiento profesional en Salud de la Mujer y Salud Mental, así como el establecimiento de un flujo asistencial adecuado en la red, garantizaría la atención integral a las mujeres embarazadas y a los recién nacidos.
\end{abstract}

Palabras clave: Enfermería; El embarazo; Alcoolismo; Síndrome de alcoolismo fetal; Salud mental.

\title{
1. Introdução
}

No Brasil, $18 \%$ das mulheres entre 18 e 24 anos, e 14\% entre 35 e 44 anos, fazem uso de álcool; sendo que uma a cada 10 está gravida (Silva, Lyra \& Diniz, 2019; Rigo et al., 2020). O abuso de álcool na gravidez acarreta uma diversidade de riscos ao bebê, como doenças do Sistema Nervoso Central, podendo levar à morte. Estes malefícios, muitas vezes, não são conhecidos pelas gestantes e nem abordados pelos profissionais da saúde durante o pré-natal (Franklin, Fernandes \& Vilela,2020).

Com maior vulnerabilidade, as mulheres precisam da metade da dose de álcool ingerida pelos homens, para se intoxicarem. Isto ocorre devido às suas características físicas, como a menor quantidade de água e maior quantidade de gordura corporal, que dificultam a ação das enzimas que metabolizam o álcool (Silva, Lyra \& Diniz, 2019). Qualquer quantidade de álcool pode trazer riscos à mãe e ao bebê, principalmente nos três primeiros meses de gestação, e o consumo pode estar associado às faltas nas consultas de pré-natal e ao ganho insuficiente de peso fetal (Popova, Lange, Probst, Gmel \& Rehm, 2017; Anjos, Quaresma \& Morais, 2020).

O uso de álcool na gravidez contribui para o desenvolvimento de problemas que afetam a saúde da mãe e filho podendo, inclusive, causar o Transtorno do Espectro Fetal do Álcool (TEAFs) ou Síndrome Alcoólica Fetal (SAF) (Popova et 
al., 2017). Esta síndrome se refere a deficiências do neurodesenvolvimento, resultando em problemas de aprendizagem, memória, atenção, funcionamento executivo, regulação do afeto, comportamento adaptativo, habilidades motoras e sociais e efeitos a longo prazo, a exemplo do aumento do risco de doenças autoimunes, osteoartrite, problemas de visão e audição, hipertensão, enxaquecas, dentre outros (May et al., 2018; Poole, Schimdt, Bocking, Bergeron \& Fortier, 2019).

As intervenções no âmbito da prevenção são a melhor maneira de impedir anormalidades ao feto (Anjos et al., 2021). O rastreio precoce cria possibilidades de acesso a serviços especializados de tratamento e alternativas de enfrentamento ao uso de álcool e outras drogas na gestação, evitando e/ou amenizando complicações maternas e neonatais.

O Programa de Atenção Integral a Saúde da Mulher (PAISM), criado em 1984, prevê ações de promoção, proteção, assistência e recuperação da saúde, em seus diferentes níveis de atenção, da básica a alta complexidade (Ministério da Saúde, 2004). Este programa incluiu ações educativas, preventivas, de diagnóstico, tratamento e recuperação na assistência à mulher em clínicas gineco-obstétricas (Ministério da Saúde, 2011). As Unidade Básicas de Saúde (UBS) são a porta de entrada dessas gestantes, onde as consultas do pré-natal ocorrem. (Ministério da Saúde, 2012b; Secretaria de Estado de Saúde de Goiás, 2019).

Como atividade privativa do enfermeiro, a consulta de enfermagem no pré-natal objetiva ofertar condições para a promoção da saúde da gestante, por meio do acolhimento, acompanhamento do pré-natal, interesse pelo seu modo de vida e identificação dos fatores de risco gestacional o mais precocemente possível (Ministério da Saúde, 2012b). Entre os fatores de risco gestacional, o uso de fumo e de substancias lícitas e ilícitas concorrem para as condições desfavoráveis para a evolução da gestação, e o cuidado de enfermagem neste processo constitui nosso objeto de estudo. Devido aos efeitos negativos à saúde, decorrente do uso pesado de álcool na saúde geral das mulheres e o risco de SAF quando grávidas, é de fundamental importância compreender o nível e os padrões de consumo de álcool pelas mulheres antes, durante e após a gravidez. (Himmelreich, Lutke \& Travi, 2017; May et al., 2018; Poole et al., 2019).

O atendimento dessas gestantes requer seu acompanhamento e sua inserção em grupos de apoio, incluindo os familiares. Este processo exige competências técnicas dos profissionais de enfermagem e habilidades para orientar e incentivar a abstinência completa ou redução de todas as drogas, embora, nos protocolos de pré-natal ou de saúde mental na Atenção Primária de Saúde, não existam recomendações a respeito do manejo da gestante usuária de crack ou cocaína, o que torna um desafio também para o profissional de saúde (Ribeiro, Giusti \& Ciosak, 2018; Peters, Cruzeiro, Bertolini, Assis, Silva \& Peres, 2020).

Pode ocorrer que a equipe da UBS necessite encaminhar a gestante para os serviços de pré-natal de alto risco e Centro de Atenção Psicossocial de Álcool e Outras Drogas (CAPS ad II, III) por meio da articulação e mobilização da rede de atendimento do município ou região. Entretanto, é importante que a gestante mantenha o vínculo com a equipe da Atenção Básica, que conhece o seu contexto familiar e social, assumindo responsabilidade conjunta sobre o cuidado (Ribeiro et al., 2018).

Cabe ao enfermeiro, juntamente com a equipe da UBS, manter a vigilância do comparecimento das gestantes nos retornos previstos, busca ativa no caso de não adesão, realizar ações educativas que abordem o ciclo gestacional, o compromisso com a saúde do bebê e possíveis condições de agravos à sua saúde (SES-GO, 2019).

Tendo em vista as atribuições dos enfermeiros ao ofertar o atendimento a estas gestantes, verifica-se a necessidade de um treinamento destes profissionais para que suas ações sejam adequadas e assegurem a qualidade da gestação do binômio mãe e filho, reduzindo as complicações obstétricas (Ferreira \& Miranda, 2016).

Considerando a questão norteadora "Quais são os conhecimentos em relação à Síndrome Alcoólica Fetal e as ações promovidas pelo enfermeiro no cuidar de gestantes usuárias de álcool? ”, lançada para a realização deste estudo, teve-se como 
objetivo identificar o conhecimento dos enfermeiros sobre a Síndrome Alcoólica Fetal (SAF), bem como as ações promovidas por estes no pré-natal de gestantes usuárias de álcool nas Unidades Básicas de Saúde.

\section{Metodologia}

Trata-se de uma pesquisa com abordagem qualitativa, de caráter descritivo e exploratório, desenvolvida em Unidades Básicas de Saúde (UBS), situadas em um município do interior de Minas Gerais. Consideramos para o estudo o método dialético por este possuir a percepção dos fenômenos e considerar os fatos dentro de um contexto social. Ademais, as contradições que emergem deste método são consideradas indícios de situações que requerem soluções (Pereira A. S. et al, 2018).

Participaram da pesquisa 11 enfermeiros das UBS com Estratégia de Saúde da Família, da área urbana do município, que atenderam aos critérios de inclusão e exclusão. Como critérios de inclusão, foram considerados enfermeiros da Atenção Básica com mais de um ano de experiência; de exclusão, enfermeiros que se encontravam ausentes por motivos de férias e licenças.

Para a realização da pesquisa foi solicitado autorização do Secretário de Saúde do município e, uma vez aprovado, foi feito contato com os enfermeiros das UBS por telefone; sendo que, para os que atenderam aos critérios de inclusão, foram agendadas as entrevistas, de acordo com suas disponibilidades.

A coleta dos dados foi realizada no período de maio a agosto de 2016, por uma aluna bolsista do PROBIC (Bolsas de Iniciação Científica, Tecnológica e Inovação), previamente treinada para a condução das entrevistas.

Utilizou-se, para a coleta de dados, um instrumento semiestruturado elaborado pelas autoras, composto por dados sociodemográficos e pelas seguintes questões: 1) Qual o seu conhecimento sobre a Síndrome Alcoólica Fetal? 2)Descreva a sua atuação no Pré-Natal de gestantes usuárias de álcool.

Realizou-se um pré-teste, a fim de verificar a confiabilidade e pertinência do instrumento aos objetivos da pesquisa. As entrevistas foram realizadas pela bolsista na sala das UBS para manter a privacidade, e teve duração média de 30 minutos. Foram gravadas por meio do smartphone, após consentimento dos participantes. No momento da entrevista, os enfermeiros foram informados sobre a natureza do estudo e, concordando com os termos propostos, leram e assinaram o Termo de Consentimento Livre e Esclarecido (TCLE), atendendo as Diretrizes e Normas Regulamentadoras de Pesquisas Envolvendo Seres Humanos (Resolução 466/12) (Ministério da Saúde, 2012a).

Com a intenção de manter o anonimato, os participantes do estudo foram identificados com a letra E, seguidos de número arábico, de acordo com a ordem da entrevista. Os dados coletados, após transcrição realizada pelas autoras, foram organizados conforme método de análise de conteúdo, por meio da análise temática, seguindo as etapas de pré-análise, exploração do material, tratamento, interpretação e elaboração das categorias significativas (Sousa \& Santos, 2020).

A pesquisa obteve parecer favorável pelo Comitê de Ética em Pesquisa da Pontifícia Universidade Católica de Minas Gerais, sob o número 1.623.820.

\section{Resultados}

Participaram do estudo 11 enfermeiros, sendo todas (100\%) do sexo feminino; cinco (45\%) apresentaram idade entre 26 e 30 anos; e, seis (54,5\%) possuíam tempo de formação de 6 a 10 anos. Quanto à pós-graduação, três (27\%) concluíram pós em Estratégia da Saúde da Família, uma (9\%) em Obstetrícia e Unidade de Terapia Intensiva (UTI) neonatal; três (27\%) possuíam Mestrado em Ciências da Saúde, uma (9\%) em Enfermagem do trabalho, uma (9\%) em UTI; e, três (37\%) não tinham pós-graduação. Após leitura exaustiva das narrativas obtidas, emergiram três categorias temáticas: Categoria Temática 1: falta conhecimento sobre a Síndrome Alcoólica Fetal; Categoria temática 2: estratégias utilizadas pelos enfermeiros no 
atendimento às gestantes usuárias de álcool; e, Categoria temática 3: dificuldades em se realizar a referência e contrarreferência na rede de atenção à saúde (RAS).

\subsection{Categoria Temática 1: falta de conhecimento sobre a Síndrome Alcoólica Fetal (SAF)}

A ausência de conhecimento sobre a temática foi justificada pela falta de ocorrência sobre a problemática, como se gestantes usuárias de álcool não frequentassem aquele dado serviço.

[...] ]aqui na Unidade Básica de Saúde a gente, dificilmente, encontra um bebê com esses sintomas. Pelo menos, eu nunca observei. A gente acompanha com puericultura e o atendimento da pediatra e do clínico todas as crianças da área de abrangência, de uma maneira geral. Eu nunca tive uma experiência com uma criança que tivesse algum problema de abstinência, ou que a mãe fosse usuária, assim, que a criança ficasse com a síndrome, que fosse falado. Pelo menos, isso eu não tenho conhecimento. (E6)

[...] Eu não tenho experiência com isso, porque já faz três anos que eu trabalho no PSF, e nestes três anos não acompanhei nenhuma criança filho de mãe usuária de álcool ou droga. (E11)

Para aqueles que reconheceram o problema, a justificativa de encaminhamento ao médico foi tema de algumas narrativas. Entenderam a problemática da Síndrome Alcoólica Fetal (SAF) como real, porém de competência do profissional médico.

[...] Na verdade, aqui no PSF, como tem o pediatra, as ações acabam sendo desenvolvidas pelo pediatra (sic). Nós, enfermeiros, não temos muita atuação, porque as crianças acabam passando direto com o pediatra e a puericultura acaba sendo feita por ele. Então aqui, quando eu vejo alguma alteração, passo para o médico pediatra, para o psicólogo, envolve a família também, mas as ações são mais desenvolvidas pelo médico. (E5)

Bem, não tivemos a vivência aqui nesta unidade, mas eu imagino que frente à síndrome, essa criança teria que ter um acompanhamento específico, que aí eu creio que o médico faria o encaminhamento. O psicólogo do NASF acompanharia não só a mãe, mas a família também. O que me vem à mente a princípio é isso. (E9)

\subsection{Categoria Temática 2: estratégias utilizadas pelos enfermeiros no atendimento à gestantes usuárias de álcool}

De acordo com os dados obtidos das participantes, as ações de enfermagem a essas gestantes seguem o protocolo do Ministério da Saúde, como realização de exames, acolhimento, exame físico, classificação de risco, controle do comparecimento das gestantes nos retornos previstos e busca ativa das gestantes faltosas:

[...]Então, a gente realiza busca ativa, os agentes domiciliares que são os primeiros a identificar a gestante no domicilio e trazem para a gente quem são, elas vêm até a gente para a primeira consulta, para pedir os exames de rotina de pré-natal, para fazer o acompanhamento de pré-natal, se elas não vêm, a gente vai atrás delas, para poder explicar a importância de aderir ao pré-natal, e aí, chegando na unidade, se a gente identifica que é usuária de álcool ou droga, a gente pede os exames de rotina e entra em contato com o hospital da zona leste que é referência para pré-natal de alto risco e ela é referenciada para o ginecologista fazer o pré-natal, que é o especialista. (E3) 
[...]As gestantes são motivadas a fazer o pré-natal, a realizar as consultas, os exames, tudo que é preconizado. A gente busca conscientizá-la sobre a importância do pré-natal e quando a gestante precisa de um encaminhamento para o serviço de alto risco a gente acaba encaminhando. Faz a busca ativa quando necessário, quando elas faltam nas consultas, ou fazem um pré-natal inadequado, a gente acaba fazendo a busca ativa também. (E4)

Percebe-se preocupação das profissionais com o acolhimento e vínculo como uma das estratégias para se estabelecer uma relação de confiança e assegurar a adesão e a continuidade do pré-natal:

[...] São poucos os casos das gestantes usuárias, mas elas existem. Elas são acompanhadas aqui pelo médico, o clínico e pela enfermeira, e, muitas vezes, são encaminhadas para rede de alto risco. Mas a gente prefere que elas fiquem aqui com a gente. A gestante usuária, a gente precisa ter mais atenção, porque algumas são usuárias, mas fazem o pré-natal corretamente. Outras não. São essas que somem mesmo, que são usuárias mesmo de drogas mais pesadas. Então essas a gente tem que ficar fazendo busca ativa, indo atrás para saber se fez exame. É bem difícil, normalmente acaba fazendo pouquíssimos exames. A gente pega a gestante e já faz para ficar pronto. A gente vai tentando assim, o mínimo que a gente consegue para a criança nascer melhor. Não difere tanto dos outros pré-natais, a gente só tem que ter mais atenção com as faltas e fazer busca ativa no caso dela não aparecer. (E1)

[...] Aqui a gente tem a referência que é o alto risco, mas, normalmente, essas gestantes usuárias a gente prefere acompanhar na unidade por questão do vínculo com elas. A gente tem o apoio dos agentes comunitários que fazem as visitas frequentes. Nós mesmas, as enfermeiras, podemos deslocar da unidade e ir procurar essas gestantes no domicílio, porque muitas vezes elas faltam nas consultas e aí fica sem atendimento. Essa busca ativa delas é constante... a gente dá uma atenção especial, que foge um pouco da rotina, da consulta agendada e marcada e tenta ir em busca dessas gestantes para que elas cumpram um pré-natal com todos os exames feitos, com um acompanhamento adequado. (E2)

A Visita Domiciliar (VD) foi citada como estratégia de busca ativa das gestantes que abandonam o pré-natal, e que representa papel essencial quando as gestantes não dão seguimento às consultas, pois permite o conhecimento da história clínica e social, fortalece o vínculo, identifica suas fragilidades e ajuda no planejamento das intervenções para incentivar a adesão ao serviço

Cabe ressaltar que, no atendimento a gestantes em abuso de álcool, aspectos subjetivos não foram em sua totalidade compreendidos, comprometendo, por conseguinte, a assistência do binômio mãe e filho. Estas evidências apareceram conforme os discursos a seguir:

[...] Primeira coisa é forçar a gestante, que a gente tem conhecimento que é usuária de drogas/álcool, a frequentar o pré-natal. Pré-natal é primordial, que é onde a gente faz o acompanhamento da gestante e do bebê, da avaliação do bebê intraútero, então isso é fundamental, e quando isso não acontece, a gente procura outros meios para evitar essa síndrome que, infelizmente, ele vai acabar tendo. (E7)

[...] A questão do tratamento dessa gestante eu acho assim que nem sempre é... Nesse período que seria um período da gente falar que ela tem que parar e que ela tem que tratar. Isso às vezes não é oferecido, mas não é o foco principal. O foco principal é que ela consiga fazer um pré-natal de qualidade para que não tenha nenhum comprometimento com a criança. E depois, quando ela as vezes tiver a criança, a gente tentar focar então nessa 
questão de parar, porque nem sempre a gente consegue. Se a gente ficar às vezes criticando muito o fato dela ser usuária a gente perde o vínculo, e deixa de fazer o pré-natal. Então a gente tem esse cuidado. (E8)

[...] Nós tivemos o caso de uma gestante usuária, que inclusive era moradora de rua, e ela foi viver em um albergue durante a gestação. Mas nós explicamos para ela a importância de que ela não fizesse uso do álcool e Crack durante a gestação, e ela teve uma grande força de vontade, e ela realmente durante a gestação não usou. Depois do parto ela teve uma recaída, mas durante toda a gestação, não. Então o bebê nasceu com um ótimo desenvolvimento e não apresentou nenhum sintoma de síndrome de abstinência. (E10)

Entretanto, uma das participantes demonstrou que, embora não haja uma rotina clara de acompanhamento das gestantes com esse perfil, buscou-se apoio junto à equipe do CAPS ad.

[...] O acompanhamento é o pré-natal de rotina da mesma forma das outras e, as que falam para a gente que estão conseguindo ficar sem fazer o uso, tudo bem. As que relatam que não conseguem, a gente encaminha para o CAPS ad, para dar um suporte para a gente. Então, como aqui ela chega na atenção básica e a gente a identifica como usuária, a gente já caracteriza como uma gestação de risco. Então essa gestante já é encaminhada para o pré-natal de risco, mas ao mesmo tempo que ela é acompanhada aqui, ela recebe as orientações, nas consultas de pré-natal, com relação às consequências do uso das drogas, justamente para evitar a síndrome dos bebês, e para que tenha uma evolução boa no pré-natal, mas tem alguma parceria com o CAPS ad. Quando a gente tem algum tipo de dúvida, ou acolhimento dessas gestantes, a gente também pede ajuda para eles nessas orientações. (E10)

\subsection{Categoria Temática 3: dificuldades em se realizar a referência e contrarreferência na rede de atenção à saúde (RAS)}

Quando a gestante apresenta algum sinal que configurem que ela seja de alto risco, é responsabilidade do médico e do enfermeiro encaminhá-la, quando necessário, para um serviço de referência. Este encaminhamento visa um acompanhamento ao pré-natal com cuidados especializados apropriados à demanda. Entretanto, segundo os dados obtidos das participantes, constatou-se que não há contrarreferência efetiva entre os serviços envolvidos.

[...] A referência é feita para o alto risco, e as vezes para o CAPS ad também. Normalmente só tem a referência, e a contrarreferência não acontece. A gente não tem a resposta, eu nunca tive nenhuma resposta assim do CAPS ad. Eu falo, isso é importante do acompanhamento que é feito na zona sul, lá a gente tem o núcleo que tem uma obstetra, que faz o acompanhamento da gestante. A zona leste tem um outro núcleo, que também tem acompanhamento lá. Lá é diferente. Assim, não pode generalizar que isso seja igual, não é igual. Lá elas são acompanhadas pela médica certinho, mas eles não têm tanta preocupação com a falta. Lá na zona leste é feito, eles ligam, eles têm contato, eles ligam para a gente, porque as vezes acontece de a gestante parar lá, por algum problema justamente. Então assim, só para vocês entenderem que tem diferença. Então a gente faz a referência, mas nunca recebe a contrarreferência dessas gestantes, e, até porque eu acho que o próprio profissional ele tem um pouco, eu acho de preguiça de investir nisso. Porque ele acha que ele vai perder tempo. Ele não vai, ele não vai ganhar a causa, é um meio que um caso perdido. Mas a gente não pode pensar assim. E uma vantagem que a gente tem aqui é que a gente tem um vínculo, se a gente fizer o vínculo com essa gestante ela vai acreditar na gente, e aí a gente consegue fazer um serviço, então as vezes encaminhar é bom para saber se a criança está bem formada, mas isso o médico daqui também pode pedir um ultrassom morfológico, pode ser bem acompanhada aqui. Se ela não estiver tendo nenhuma alteração assim, de 
pressão, nenhuma alteração física, de sintoma, dá para ser acompanhada bem aqui, até melhor, porque a gente tem o vínculo e consegue assim, tirar mais coisa dela, e consegue convencer ela de mais coisa. (E4)

[...] A gente entra em contato com o Programa de Saúde da Mulher e manda uma, como é que eu vou te explicar, uma referência e uma contrarreferência para lá, eles estudam o caso e eles entram em contato com a gestante e já chama se for algum caso mais grave. A gente entra em contato por telefone mesmo e explica a necessidade de uma consulta mais rápida, e aí o acompanhamento é feito lá. Quando ela começa a faltar em consulta, a dar muito problema, é acompanhado nos dois lugares, aí a enfermeira responsável do Programa de Saúde da Mulher me liga para me falar como é que está sendo o acompanhamento lá para a gente fazer o acompanhamento pelas duas unidades, em caso de falta, em caso dela começar a dar muito trabalho. (E7)

[...] Aqui a gente tem como referenciar as gestantes para o alto risco, que aí tem uma equipe para tratar de médicos e enfermeiros, mas nem sempre a gente dá essa opção porque às vezes encaminhar a gente não tem como saber se ela foi ou não, e também tem que referenciar elas para o tratamento de álcool, que é o CAPS ad, que tem uma parceria legal, as vezes a gente liga para eles, eles vêm, fazem visita com a gente, é uma possibilidade às vezes a gente entrar em contato com eles. Eu já consegui fazer algumas visitas em conjunto, com eles. Porque no caso mandar essas pessoas para o serviço de referência e esperar que elas vão, nem sempre é a melhor solução, e assim nem sempre há a contrarreferência desses serviços, principalmente, da questão do pré-natal de alto risco. A gente não tem muito, infelizmente, isso é um defeito do sistema, uma coisa a se melhorar, a gente não tem contrarreferência, se ela está indo, se ela não está indo, se ela faltou, se não faltou, então, assim, do CAPS ad é até possível conseguir, mas da gestante e do alto risco não. Então assim, igual eu falei, vamos focar no pré-natal, as vezes nós mesmos, com o que a gente tem na unidade, a gente foca para depois tentar esse tratamento, né. (E10)

\section{Discussão}

A atuação dos enfermeiros na realização da consulta pré-natal de gestantes em abuso de álcool é um desafio que se mostra a cada dia mais complexo, e requer desses profissionais uma revisão da prática para garantir às gestantes um atendimento seguro. Deve oferecer condições para a promoção da saúde da gestante e a melhoria na sua qualidade de vida, onde as informações passadas sobre os efeitos do álcool e droga no feto são de extrema importância (Capeletti, Lins \& Giotto, 2019).

Estudos recentes consideram importante conhecer o perfil das gestantes, para que o nascimento dos bebês com SAF possa ser acompanhado num esforço direcionado de prevenção e intervenção; além disso, mostram que as investigações anteriores focavam apenas populações vulneráveis de baixo e alto risco socioeconômico e escolaridade, com níveis elevados de consumo de substâncias químicas entre seus parceiros e famílias. Entretanto, resultados atuais têm evidenciado que o consumo tem se elevado em população de alta renda e dados demográficos diversificados, denunciando que o consumo de álcool cresceu em mulheres de todas as classes sociais, durante a gravidez. (Popova et al., 2020)

As ações de educação em saúde, componente fundamental do trabalho técnico voltado à saúde materno-infantil, precisam estar claras na prática profissional do enfermeiro, especialmente no que se refere ao consumo de substância lícitas e ilícitas. O momento da consulta de enfermagem no pré-natal é oportuno para discutir os malefícios do uso do álcool, e como se reflete no organismo materno-fetal em decorrência do consumo (Sousa, Sales, Oliveira \& Chagas, 2018; Peters et al., 2020).

Para tanto, sugere-se que o profissional de enfermagem seja bem qualificado para atender às especificidades do cuidado às gestantes com esse perfil, que seja capaz de promover ações de educação em saúde que levem conhecimento tanto às gestantes quanto aos seus parceiros e à comunidade em geral (Sousa et al., 2018). Não obstante, instituído pelo Ministério 
da Saúde, é realizado o pré-natal do parceiro, que busca contextualizar a importância do envolvimento consciente e ativo do companheiro em todas as ações voltadas ao planejamento reprodutivo e estimular a sua participação durante o processo do prénatal, para o bem-estar biopsicossocial da mãe, do bebê e dele próprio (Ministério da Saúde, 2018).

Para atender as gestantes que abusam do álcool, o conhecimento do profissional deve ir além do protocolo determinado pelo Ministério da Saúde no atendimento de baixo e alto risco. Deve incluir competências e habilidades no cuidado, em torno dos riscos do uso de substâncias químicas na gestação, com o objetivo de promover a saúde e prevenir agravos, o que constitui um grande esforço profissional (Peters et al., 2020).

Pelas informações obtidas neste estudo, verificou-se que as enfermeiras têm um conhecimento vago sobre a SAF. Resultado semelhante em outro estudo trouxe que o conhecimento limitado sobre a SAF, ou os efeitos do uso do álcool durante a gravidez, esteve presente entre os enfermeiros; alguns desconheciam as manifestações da síndrome ou não estavam cientes dos riscos específicos do consumo de álcool na gestação, o que demonstra uma lacuna nos cursos de graduação; principalmente porque, ao ministrar a disciplina de Enfermagem em Saúde Mental, não é abordada a SAF, como tema relacionado ao estudo do abuso de substâncias psicoativas (Santos, Estefanio \& Figueiredo, 2017).

Considera-se, ainda, uma falha do Ministério da Saúde em não divulgar amplamente campanhas educativas sobre a SAF, em subnotificar a síndrome e em não ofertar cursos de capacitação para os profissionais que trabalham na Atenção Básica de Saúde, especialmente com o pré-natal de gestantes em uso de substâncias lícitas e ilícitas, dada a necessidade de compreenderem melhor as singularidades de cada mulher com este perfil, para garantir a qualidade da assistência ofertada a elas (Peters et al., 2020).

As equipes das UBS devem ser preparadas para desenvolver as aptidões essenciais na atenção no pré-natal, para se identificar as gestantes de risco, logo no início da gravidez, desenvolver atitudes de sensibilidade, aceitação e empatia; saber ouvir, ter uma interação afetiva para facilitar o vínculo e um conhecimento básico acerca da doença mental e dos modelos terapêuticos que os habilite a planejar as atividades assistenciais e os procedimentos habituais (Marcolino et al., 2018).

Quando a equipe cuidadora está preparada, ajuda a despertar o desejo das mães em não causar malefícios na saúde do seu bebê; e é nesta fase que se consegue uma abstinência completa e duradoura de todas as drogas (Ministério da Saúde, 2012b). Dessa maneira, o diagnóstico e as intervenções de enfermagem no pré-natal, visando a abstinência ou redução do consumo dessas substâncias, devem constituir uma atitude prioritária na atenção básica para essa população. O pré-natal deve incluir, além da realização das consultas clínicas obstétricas, atividades educativas indispensáveis para divulgar o problema e orientá-las sobre as complicações para si, para o feto e recém-nascido (Lemos \& Taveira, 2016).

Para programar as atividades de controle do pré-natal, é necessário identificar os riscos aos quais cada gestante está exposta, de acordo com a droga e a frequência de uso. É indispensável que essa avaliação do risco seja permanente e aconteça em toda consulta, para garantir os direitos do binômio mãe-filho (Murta, Guimarães, Martins \& Cunha, 2018).

Essas ações educativas podem ser realizadas em grupos que proporcionam oportunidades para a discussão do tema e esclarecimento de suas dúvidas. Favorecem o vínculo, a confiança, reciprocidade entre elas; cultiva-se a existência de espaços de escuta, reflexão e empatia, de modo que se sintam acolhidas e favoreça a adesão às orientações nas consultas (Peters et al., 2020).

Os grupos compõem um importante cenário para abordar questões relacionadas à maternidade e são um espaço fértil para a construção da relação materno-fetal, valorização da maternidade como parte do caminho para o enfrentamento da dependência química (Ribeiro, Rocha, Bragé, Ramos \& Vrech, 2020).

Neste estudo, as participantes citaram o encaminhamento das gestantes em uso de bebida alcoólica para serviços que atendam, especificamente, gestações de alto risco; e apenas duas mencionaram o apoio e o encaminhamento da gestante para um serviço especializado em álcool e outras drogas (CAPS ad). Destacaram a falta de articulação entre os dispositivos da Rede 
de Atenção à Saúde (RAS), mencionando a perda de contato com as gestantes, desconhecimento dos procedimentos realizados nos serviços referenciados e da frequência delas no serviço de alto risco e CAPS ad.

As RASs são conjuntos de serviços de saúde vinculados entre si, que ofertam atenção contínua, integral e humanizada para determinada população, coordenados pela Atenção Primária à Saúde, sendo importante a articulação entre os serviços de saúde para que os trabalhadores possam estabelecer comunicações e trocas, a fim de criar vínculo com o usuário e manter a continuidade de sua assistência, fazendo com que se efetivem os processos de referência e contrarreferência (Brondani, Leal, Potter, Silva, Noal \& Perrando, 2016).

Um dos desafios para o cuidado integral é a fragmentação da RAS e o desconhecimento dos trabalhadores sobre o funcionamento da rede. E, em decorrência disso, a atenção básica não consegue exercer sua principal função, que é coordenar o cuidado (Brondani et al., 2016). Para que se obtenha uma rede articulada, e os processos de referência e contratransferência se efetivem, é necessário, um planejamento e criação de fluxo municipal sobre esse processo, bem como uma mudança de atitude para trabalhar de forma sistematizada, sendo muitas vezes necessária o uso da educação permanente da equipe multiprofissional.

O CAPS ad, por sua vez, é um local aberto, territorializado e específico, devido sua concepção ampliada, para desenvolver estratégias de enfrentamento para a abstinência ou redução de danos em relação ao uso de álcool, de prevenção, recuperação e reinserção social do indivíduo. O atendimento pela equipe, tanto individual como grupal, possibilita a expressão dos sentimentos, ansiedades, angústias, proteção nos momentos de crise e tenta desenvolver a corresponsabilização da gestante pela sua saúde e do seu filho (Machado, Moderna \& Luz, 2020). Quando o atendimento à gestante em uso de álcool é desarticulado entre os enfermeiros das UBS, dos serviços de pré-natal de alto risco e do CAPS ad, aumenta a probabilidade de insucessos na realização de um pré-natal seguro. Essa desarticulação é prejudicial para o binômio mãe-filho, aumentando os casos de SAF na população.

\section{Limitação do estudo}

Um fator limitante do estudo é de ter definido uma única categoria profissional, não compreendendo as demais especialidades das Unidades Básicas responsáveis pelo pré-natal, para conhecer como é o atendimento de cada profissional, como se articulam entre si diante das dificuldades com a gestante, pois a contribuição de cada um fortalece as ações e dá suporte à gestante. Por ser tratar de estudo com elementos de uma realidade específica, não se objetiva fazer generalizações, as propostas a partir dos resultados visam reflexões sobre uma temática atual e cada vez mais crescente em nosso país.

\section{Considerações Finais}

Considera-se, a partir dos achados, que os enfermeiros possuem pouco conhecimento sobre a Síndrome Alcoólica Fetal durante o pré-natal de gestantes usuárias de álcool nas Unidades Básicas de Saúde. Este comportamento acarreta atitudes como encaminhamentos para outros serviços e profissionais, podendo ocasionar a desvinculação da gestante com a UBS pertencente ao seu território. Não obstante, as ações dos enfermeiros devem estar permeadas por estratégias que abordem a temática álcool e gravidez, enfatizando os efeitos teratogênicos causados pela ingestão durante a gestação. Estes assuntos poderão ser abordados em espaços de discussões e programas de educação permanente.

Dentre as estratégias e ações que mais permearam as ações dos enfermeiros com relação às gestantes usuárias de álcool, foram priorizadas as visitas domiciliárias e a busca ativa em outros espaços de convivência, o foco principal desta ação foi a de conscientizar as gestantes sobre a necessidade em comparecerem durante as consultas de pré-natal, serem mais bem avaliadas e, muitas vezes, encaminhadas a outros serviços. 
Sobre as dificuldades trazidas pelos enfermeiros com relação à temática, a que mais impacta o processo assistencial é a inexistência de fluxo pactuado que respeite as obrigações pertinentes a referência e contrarrefência de rede de atenção à saúde. Este estudo contribui para reflexão sobre a normatização do fluxo das gestantes na rede e, no caso de encaminhamento para serviços especializados em situações de risco e vulnerabilidades, faz-se necessário a articulação do enfermeiro com os serviços de referência, para garantir o acompanhamento contínuo e monitorar as condições em que a gravidez ocorre.

\section{Referências}

Anjos, P. M., Quaresma, V. V. A. \& Morais, Y. J. (2020). Effects of alcohol ingestion in the management period. Revista Acadêmica online. http://files.revista-academica-online.webnode.com/200000696-a483da483f/Aricient0009162020.pdf

Brondani, J. E., Leal, F. Z., Potter, C., Silva, R. M., Noal, H.C. \& Perrando, M. S. (2016). Desafios da referência e contrarreferência na atenção em saúde na perspectiva dos trabalhadores. Cogitare Enfermagem, 21(1), 01-08.

Capeletti, A. O., Lins, J. P. \& Giotto, A. C. (2019). The interventions of nursing professionals in relation to pregnant women using licit and illicit drugs. Revista de Iniciação Científica e Extensão, 2(Esp.2), 323-8.

Ferreira, B. R. F. \& Miranda, J. K. S. (2016). As complicações causadas pelo consumo de drogas lícitas e ilícitas durante a gestação: um desafio para a equipe de enfermagem. Revista Científica de Enfermagem, 6(18), 36-43.

Franklin, T. A., Fernandes, J. D. \& Vilela, A. B. A. (2020). Analysis of scientific production about fetal alcoholic syndrome and their relationship with the health of children. Research, Society and Development, 9(9), e141997143.

Himmelreich, M., Lutke, C. \& Travis, E. (2017, março). Lay of the Land: Final Results of a Health Survey of 500+ Adults Diagnosed with FASD. In Proceedings of the 7th International Conference on Fetal Alcohol Spectrum Disorder, Vancouver, BC, Canada.

Lemos, I. R. O. \& Taveira, G. M. T. (2016). Prenatal conditions of pregnant women users legal drugs that calved in reference hospital in Singapore, 20132014. Revista Portal Saúde e Sociedade, 1(2), 130-145.

Machado, A. R., Moderna, C. M. \& Luz, Z. M. P. (2020). Das proposições da política às práticas dos serviços: há novidades nos Centros de Atenção Psicossocial Álcool e Drogas? Physis: Revista de Saúde Coletiva, 30(11), e300118.

Marcolino, T. Q., Joaquim, R. H. V. T., Wernet, M., Giovanetti, G., Bongiovanni, K., et al. (2018). Gestação e uso de substâncias psicoativas: qual é o cuidado em saúde desejado pelas mulheres?. Caderno de Saúde Coletiva., 26(3), 255-260.

May, P. A., Chambers, C. D., Kalberg, W. O., Zellner, J., Feldman, H., Buckley, D., et al .(2018). Prevalence of Fetal Alcohol Spectrum disorders in 4 US communities. JAMA, 319(5), 474-482.

Ministério da Saúde. (2004). Política nacional de atenção integral à saúde da mulher: princípios e diretrizes. Secretaria de Atenção à Saúde. Departamento de Ações Programáticas Estratégicas. Brasília, DF: Ministério da Saúde.

Ministério da Saúde (2011). Portaria no 1459 de 24 de junho de 2011. Institui, no âmbito do Sistema Único de Saúde - SUS - a Rede Cegonha. Brasília, DF. Ministério da Saúde.

Ministério da Saúde (2012a). Resolução no 466 de 12 de dezembro de 2012: aprova as diretrizes e normas regulamentadoras de pesquisa envolvendo seres humanos. Conselho Nacional de Saúde, Comissão Nacional de Ética em Pesquisa. Brasília, DF: Ministério da Saúde.

Ministério da Saúde (2012b). Atenção ao pré-natal de baixo risco. Secretaria de Atenção à Saúde. Departamento de Atenção Básica. Brasília: DF. Ministério da Saúde.

Ministério da Saúde (2018). Guia do pré-natal do parceiro para profissionais de Saúde. Secretaria de Atenção à Saúde. Departamento de Ações Programáticas Estratégicas. Brasília, DF: Ministério da Saúde.

Murta, N. N. R., Guimarães, C. F. B., Martins, T. M. D. \& Cunha, R. G. (2018). Perfil gestacional e exposição fetal a drogas de abuso. Revista Núcleo de Biociências, 8(16), 74-87.

Pereira A. S. et al. (2018). Metodologia da pesquisa científica. [free e-book]. Santa Maria/RS. Ed. UAB/NTE/UFSM.

Peters, A. A., Cruzeiro, H. R., Bertolini, O. G. P., Assis, G. P., Silva, A. D. \& Peres, M. A. A. (2020). Pregnant women using psychoactive substances attended by nurses in Primary Health Care. Revista Eletrônica Saúde Mental Álcool e Drogas, 16(2), 66-74.

Poole, N., Schimdt, R. A., Bocking, A., Bergeron, J. \& Fortier, I. (2019). The potential for Fetal Alcohol Spectrum disorder prevention of a harmonized approach to data collection about alcohol use in pregnancy cohort studies. International Journal of Environmental and Public Health, 16(11).

Popova, S., Lange, S., Probst ,C., Gmel, G. \& Rehm, J. (2017). Estimation of national, regional, and global prevalence of alcohol use during pregnancy and fetal alcohol syndrome:a systematic review and meta-analysis. The Lancet Global Health, 5, e290-99.

Popova, S., Lange, S., Temple, V., Poznyak, V., Chudley, A. E., Burd, L., et al. (2020). Profile of mothers of children with Fetal Alcohol Spectrum disorder: a population-based study in Canada. International Journal of Environmental and Public Health, 17, 7986.

Ribeiro, L. S., Rocha, D. G., Bragé, E. G., Ramos, D. B. \& Vrech, L. R. (2020). Enfrentamento da dependência química na gestação por meio de grupos terapêuticos. The Brazilian Journal of Health Review, 3(2), 1437-1445. 
Research, Society and Development, v. 10, n. 9, e0710917717, 2021

(CC BY 4.0) | ISSN 2525-3409 | DOI: http://dx.doi.org/10.33448/rsd-v10i9.17717

Ribeiro, M. C. L., Giusti, B. B. \& Ciosak, S. I. (2018). Cuidado de mulheres usuárias de crack na gestação: revisão bibliográfica. Revista Eletrônica Saúde Mental Álcool e Drogas, 14(3), 185-193.

Rigo, F. L., Prates, M. L., Camponezês, P. S. P., Silveira, T. V. L., Costa, R. P. G., Cunha, A. C. C., et al. (2020). Prevalência e fatores associados ao uso de álcool, tabaco e outras drogas em gestantes. Revista Médica de Minas Gerais, 30, e-30117.

Santos, R. S., Estefanio, M. P. \& Figueiredo, R. M. (2017). Prevention of fetal alcohol syndrome: input to obstetrical nurses' practice. Revista Enfermagem UERJ, 25, e277993.

Secretaria de Estado de Saúde - Goiás (2019). Pré-natal. Goiás: Secretaria do Estado de Saúde. Disponível em: https://www.saude.go.gov.br/biblioteca/7637pr\%C3\%A9-natal.

Silva, M. G. B., Lyra, T. M. \& Diniz, G. T. (2019). The pattern of alcohol consumption among the users of the Family Health Units in the municipality of Recife (PE). Saúde Debate, 43(122), 836-847.

Sousa, D. M. O., Sales, F. A. G., Oliveira, J. H. M. \& Chagas, A. C. F. (2018). Caracterização das gestantes de alto risco atendidas em um centro de atendimento à mulher e o papel do enfermeiro nesse período. Revista Atenção à Saúde, 16(56), 54-62.

Sousa, J. R. \& Santos, S. C. M. (2020). Análise de conteúdo em pesquisa qualitativa: modo de pensar e de fazer. Pesquisa e Debate em Educação,10(2), 1396-1416. 